\title{
Managing incidental pancreatic cystic neoplasms with integrated molecular pathology is a cost-effective strategy
}

Authors

Institutions
Ananya Das ${ }^{1}$, William Brugge ${ }^{2}$, Girish Mishra ${ }^{3}$, Dennis M. Smith ${ }^{4}$ Mankanwal Sachdev ${ }^{1}$, Eric Ellsworth ${ }^{4}$

${ }^{1}$ Arizona Center for Digestive Health, Gilbert, Arizona, United States

${ }^{2}$ Digestive Healthcare Center, Massachusetts General Hospital, Boston, Massachusetts, United States

${ }^{3}$ Department of Gastroenterology, Wake Forest Baptist Health, Winston-Salem, North Carolina, United States

${ }^{4}$ RedPath Integrated Pathology, Pittsburgh, Pennsylvania, United States submitted

13. February 2015

accepted after revision

2. March 2015

\section{Bibliography}

DOI http://dx.doi.org/

10.1055/s-0034-1392016

Published online: 26.6.2015

Endosc Int Open 2015; 03:

E479-E486

(c) Georg Thieme Verlag KG

Stuttgart · New York

E-ISSN 2196-9736

\section{Corresponding author}

\section{Ananya Das, MD}

Arizona Center for Digestive Health

2680 S Valvista Drive, Suite \#116

Gilbert, Arizona 85295

United States

Fax: +1-480-507-5677

adas@azcdh.com
Background and study aims: Current guidelines recommend using endoscopic ultrasound (EUS), carcinoembryonic antigen (CEA) testing and cytology to manage incidental pancreatic cystic neoplasms (PCN); however, studies suggest a strategy including integrated molecular pathology (IMP) of cyst fluid may further aid in predicting risk of malignancy. Here, we evaluate several strategies for diagnosing and managing asymptomatic PCN using healthcare economic modeling. Patients and methods: A third-party-payer perspective Markov decision model examined four management strategies in a hypothetical cohort of 1000 asymptomatic patients incidentally found to have a $3 \mathrm{~cm}$ solitary pancreatic cystic lesion. Strategy I used cross-sectional imaging, recommended surgery only if symptoms or risk factors emerged. Strategy II considered patients for resection without initial EUS.Strategy III (EUS+ CEA+Cytology) referred only those with muci-

$\begin{array}{ll}\text { Abbreviations } \\ \text { ACG } & \text { American College of Gastroenterology } \\ \text { ASA } & \text { American Society of Anesthesiology } \\ \text { CEA } & \text { carcinoembryonic antigen } \\ \text { CT } & \text { computed tomography } \\ \text { EUS } & \text { endoscopic ultrasound } \\ \text { FNA } & \text { fine needle aspiration } \\ \text { ICER } & \text { Incremental Cost-Effectiveness Ratio } \\ \text { IMP } & \text { integrated molecular pathology } \\ \text { INHB } & \text { Incremental Net Health Benefit } \\ \text { IPMN } & \text { intraductal papillary mucinous neoplasms } \\ \text { LOH } & \text { loss of heterozygosity } \\ \text { MCN } & \text { mucinous cystic neoplasms } \\ \text { NHB } & \text { Net Health Benefit } \\ \text { NNT } & \text { Number Needed to Treat } \\ \text { PCN } & \text { pancreatic cystic neoplasms } \\ \text { PFTG } & \text { PathFinder TG } \\ \text { QALY } & \text { quality-adjusted life-years } \\ \text { SCN } & \text { serous cystic neoplasms } \\ \text { WTP } & \text { Willingness to Pay }\end{array}$

nous cysts $(C E A>192 \mathrm{ng} / \mathrm{mL})$ for resection. Strategy IV implemented IMP; a commercially available panel provided a "Benign," "Mucinous," or "Aggressive" classification based on the level of mutational change in cyst fluid. "Benign" and "Mucinous" patients were followed with surveillance; "Aggressive" patients were referred for resection. Quality-adjusted life-years (QALY), relative risk with $95 \% \mathrm{CI}$, Number Needed to Treat (NNT), and incremental cost-effectiveness ratios were calculated.

Results: Strategy IV provided the greatest increase in QALY at nearly identical cost to the cheapest approach, Strategy I. Relative risk of malignancy compared to the current standard of care and nearest competing strategy, Strategy III, was 0.18 (95\%CI $0.06-0.53)$ with an NNT of $56(95 \%$ CI 34 -120).

Conclusions: Use of IMP was the most cost-effective strategy, supporting its routine clinical use.

\section{Introduction}

In clinical practice, pancreatic cystic neoplasms (PCN) are being increasingly discovered on abdominal imaging studies performed for unrelated indications. Over $90 \%$ of incidental PCNs can be broadly categorized as mucinous or non-mucinous [1,2] Differentiating between these two cyst types is important because non-mucinous are commonly benign without risk of malignancy, while mucinous are considered to have an appreciable, although low, risk of malignant transformation [3]. Imaging and endoscopic features readily distinguish most serous cystadenomas (SCN) and main duct intraductal papillary mucinous neoplasms (IPMN) from other types of lesions. However, although side-branch IPMN and mucinous cystic neoplasms (MCN) are distinct histopathological entities, imaging alone often cannot definitively differentiate them. 


\begin{tabular}{l} 
Strategy I: Conservative "wait \& watch" \\
Follow all patients, refer for resection only if symptoms occur or \\
warrisome features appear \\
\hline Strategy II: Resect if operable \\
Refer all cysts for resection immediately, no followup if patient is inoperable \\
\hline Strategy III: Risk-stratify by EUS + FNA/CEA \& cytology \\
Use CEA \& cytology to differentiate mucinous from non-mucinous cysts \\
- Mucinous: Refer for resection \\
- Non-Mucinous: Follow annually for first 3 years, every $3^{\text {rd }}$ years \\
thereafter \\
\hline Strategy IV: Risk-stratify by EUS + FNA/CEA \& cytology \& IMP \\
- Non-Mucinous: Follow annually for first 3 years, every 3 rd years \\
thereafter \\
- Mucinous/Benign: Follow annually for first 3 years, every 3 years \\
thereafter \\
- Mucinous/Indolent: Follow annually for first 5 years, every 3 years \\
thereafter \\
- Mucinous/Aggressive: Refer for resection
\end{tabular}

Fig. 1 Summary of the four patient management strategies evaluated in the model. Further details can be found in the Methods section under Strategies, and in Table $\mathbf{S 2}$ of the supplement.

The risk progression to cancer is a primary concern. Although surgery is effective in preventing cancer, many of PCNs are discovered in elderly patients with concomitant co-morbidities for whom aggressive prophylactic surgery is contraindicated [4]. The American College of Gastroenterology's (ACG) 2007 practice guidelines for managing PCN recommend EUS-FNA and analysis of cyst fluid for tumor markers and cytology [5]. Per these guidelines, choosing surgery over surveillance should be based on whether the cyst is mucinous along with the presence or absence of additional risk factors (e.g., increasing cyst size, presence of mural nodules, solid component). In a previous analysis, we examined Strategies I- III, studied here, and found the most cost-effective approach to stratifying a patient's risk of developing cancer from a cyst was to use EUS-FNA and cyst fluid analysis for CEA estimation [6].

Because morphological features of cysts lack strong predictive accuracy for malignancy, patients are often managed with surgery to mitigate the possibility of progression to cancer. Many resected cysts are benign, causing both unnecessary morbidity for patients and excessive costs to the healthcare system [4,7]. Recent studies have shown that integrated molecular pathology (IMP) of patient cyst fluid may improve the ability to distinguish mucinous from non-mucinous cysts and is particularly helpful in predicting cysts' malignant potential $[8,11]$.

IMP of cyst fluid for determining malignant potential was validated in the National Pancreatic Cyst Registry, which included clinical and molecular data from 492 patients who had IMP testing of pancreatic cyst fluid (PathFinder TG, RedPath Integrated Pathology, Pittsburgh, Pennsylvania, United States) as part of usual care. The registry findings support the use of IMP to augment first-line testing in determining a course of treatment based on the likelihood of developing malignancy. With a negative predictive value of $97.2 \%$, IMP can reliably identify cysts that will not develop malignancy, thereby reducing unnecessary surgeries and their related morbidity and mortality [12].

While data produced by IMP are promising, molecular analytical techniques are more expensive than other diagnostic modalities. In lieu of a prospective, randomized clinical trial, this study used healthcare economic modeling to evaluate the costs and benefits of different strategies for diagnosing and managing asymptomatic PCN.

\section{Patients/material and methods}

$\nabla$

We simulated a hypothetical cohort of 1000 asymptomatic patients incidentally found to have a $3 \mathrm{~cm}$ solitary PCN on cross-sectional imaging. For baseline analysis, each patient was assumed to have an American Society of Anesthesiology (ASA) score of III. The model evaluated the impact of the cysts over the patients' lifetime.

\section{Model strategies}

The model compared four management strategies ( $\bullet$ Fig. 1):

I. Wait \& Watch, conservative: Cysts were followed using crosssectional imaging and surgical consultation for resection of the cyst occurred only if the patient developed symptoms or high-risk morphological features.

II. Resect if operable, aggressive: All patients were referred for surgical consultation for cyst resection. Operability was determined according to a surgical risk score as described below. No EUS-FNA was performed.

III. EUS + CEA+Cytology, standard of care: After cross-sectional imaging, all patients underwent an EUS-guided FNA for differentiating between mucinous and non-mucinous cysts. Patients diagnosed with a mucinous cyst (e.g., via cytology or elevated CEA) were referred for surgical resection. Those with a non-mucinous diagnosis were followed in the model with periodic imaging surveillance as described below.

IV. EUS + CEA + Cytology + IMP: All patients initially underwent first-line testing, as in Strategy III, followed by molecular testing. PathFinder TG IMP incorporates first-line testing results with findings from molecular testing. Patients diagnosed as "Benign" via IMP were followed as in Strategy I, while those diagnosed as "Statistically Indolent" underwent more frequent surveillance. Those diagnosed as "Aggressive" were referred for surgical resection.

\section{Surveillance and surgery in the model}

Using decision analysis software (TreeAge Pro, TreeAge Software, Inc, Williamstown, Massachusetts, United States), we built a hybrid model of a linear decision tree terminating in a Markov model. The Markov component of the model simulates the natural history of each patient's PCN using various health and disease states plus cancer related mortality. The varying malignant potential of mucinous cysts was considered. To account for age and gender-specific annual mortality from all other causes, the model incorporates US life table mortality rates.

SCN are typically identifiable on cross-sectional imaging [5], and these cysts were considered to have a benign course. Because mucinous cysts have varying malignant potential, the model incorporated both the probability of malignancy upon presentation and malignancy developing over time, and relevant performance characteristics for diagnosing mucinous vs. non-mucinous and malignant vs. benign ( Table 1, Table S1).

Patients determined to have mucinous cysts underwent surveillance with cross-sectional imaging annually for 3 years and every third year thereafter. Any patient who developed symptoms or high-risk morphological features had their surveillance interval cut in half until the symptoms resolved, malignancy emerged, or surgical resection was performed. Patients with mucinous cysts classified as "Indolent" using IMP were followed annually for 5 years and every third year thereafter. Pancreatic cystic neoplasm, specifically side-branch IPMN is a multifocal disease and most patients may be potential candidates for continued surveillance 
Table 1 Estimates for model variables (supporting references are noted in Table S1 of the Supplement).

\begin{tabular}{|c|c|c|c|}
\hline Model variable & $\begin{array}{l}\text { Strategies that use } \\
\text { this variable }\end{array}$ & $\begin{array}{l}\text { Baseline } \\
\text { value }\end{array}$ & $\begin{array}{l}\text { Range for sensitivity } \\
\text { analyses }\end{array}$ \\
\hline \multicolumn{4}{|l|}{ Development of malignancy (\%) } \\
\hline Cystic lesions that are non-mucinous (e. g., serous cystadenoma, pseudocyst) & All & 30 & $10-60$ \\
\hline Biological aggressiveness of mucinous cysts/ branch type IPMN (at presentation) & All & & \\
\hline Benign & & 65 & $0-100$ \\
\hline Borderline/indolent & & 20 & $0-100$ \\
\hline Malignant & & 15 & $0-100$ \\
\hline $\begin{array}{l}\text { Probability of asymptomatic mucinous cyst or side-branch IPMN becoming symptomatic } \\
\text { (annual) }\end{array}$ & All & & \\
\hline Cyst is $\leq 3 \mathrm{~cm}$ & & 2 & $0-5$ \\
\hline Cyst is $>3 \mathrm{~cm}$ & & 10 & $1-15$ \\
\hline \multicolumn{4}{|l|}{$\begin{array}{l}\text { Probability of benign mucinous cystic lesion/branch type IPMN transitioning from benign } \\
\text { to malignant (years) }\end{array}$} \\
\hline Cyst is $\leq 3 \mathrm{~cm}$ & & 2.5 & $0-50$ \\
\hline Cyst is $>3 \mathrm{~cm}$ & & 5 & $0-50$ \\
\hline Probability of malignant cysts becoming symptomatic (annual) & & 25 & $0-100$ \\
\hline \multicolumn{4}{|l|}{ Performance characteristics of diagnostic tests (\%) } \\
\hline \multicolumn{4}{|l|}{ Differentiating mucinous from non-mucinous cysts } \\
\hline MRI/CT (sensitivity) & All & 70 & $50-100$ \\
\hline CEA + cytology (sensitivity) & III, IV & 80 & $50-100$ \\
\hline CEA + cytology (specificity) & III, IV & 65 & $0-80$ \\
\hline PathFinder TG + CEA + cytology (sensitivity) & IV & 68 & $50-80$ \\
\hline PathFinder TG + CEA + cytology (specificity) & IV & 90 & $70-95$ \\
\hline \multicolumn{4}{|l|}{ Distinguishing aggressive from non-aggressive cysts } \\
\hline PathFinder sensitivity & IV & 82 & $70-90$ \\
\hline PathFinder specificity & IV & 85 & $70-90$ \\
\hline \multicolumn{4}{|l|}{ Mortality and utility (used in calculating QALY) } \\
\hline Perioperative mortality (years) & & 3 & $1-15$ \\
\hline Mortality from invasive malignant cysts (years) & & 10 & $0-5$ \\
\hline Normal (\%) & & 1.0 & $(\mathrm{~N} / \mathrm{A})$ \\
\hline Incidental cyst (\%) & & 1.0 & $0.75-1$ \\
\hline Symptomatic cyst (\%) & & 0.95 & $0.7-1$ \\
\hline Postoperative state (\%) & & 0.95 & $0.7-1$ \\
\hline Early cancer (\%) & & 0.9 & $0.68-1$ \\
\hline Advanced cancer (\%) & & 0.5 & $0.38-1$ \\
\hline \multicolumn{4}{|l|}{ Costs $(\$)$} \\
\hline Cross-sectional imaging (CT/MRI) & All & 1000 & $( \pm 250)$ \\
\hline EUS-FNA (including cost of sedation with monitored anesthesia care + CEA+ cytology) & III, IV & 1525 & $675-2675$ \\
\hline Pancreatic surgery & & 40000 & $( \pm 10000)$ \\
\hline Treatment for advanced malignancy - annual (e. g., chemotherapy and palliative care) & & 50000 & $( \pm 12500)$ \\
\hline PathFinder TG testing & IV & 3100 & $2500-5000$ \\
\hline Discount rate (\%) (Correction for inflation/cost increases) & & 3 & $0-7$ \\
\hline
\end{tabular}

after surgery and in the model post-surgical surveillance was allowed as in the wait and watch strategy (cross-sectional imaging every 3 years). See Table 22 in the supplement for further details. Because PCNs occur most frequently in the elderly and co-morbidities are common, a previously published operative scoring system was used to simulate whether a patient referred for surgical consultation would undergo pancreatic surgery. The scoring system has four components: patient age $(<65,65-79$, and $\geq 80$ years), surgical risk in terms of ASA score [13], cyst size $(\leq 3 \mathrm{~cm}$, $4-5 \mathrm{~cm}$, and $>5 \mathrm{~cm}$ ), and cyst location (tail, body and head of the pancreas). All patients referred for surgery were scored with this system to determine whether surgery occurred. Patients who underwent surgical resection were considered to be cured and did not undergo any further surveillance.

Analysis of the results was conducted according to the recommendations of the panel on Cost-Effectiveness in Health and Medicine for conducting and reporting a reference case analysis with a societal perspective [6]. Additional details of the decision model, strategies compared, outcome measures and, importantly, assumptions are described in previous work and in the supplement [6].

\section{Integrated molecular pathology (PathFinder TG)}

PathFinder TG uses a proprietary amplification technology to provide a full mutational analysis on aspirate fluids from the free (or released) DNA in cyst fluid specimens. The molecular analyses include three tests: k-ras gene point mutation, loss of heterozygosity $(\mathrm{LOH})$ analysis using a preselected panel of 15 genomic loci associated with tumor suppressor genes, and determination of DNA quantity/quality in cyst fluid. Each of the three tests is defined as "abnormal" when the following are identified: 1) k-ras gene point mutation, 2) LOH mutations in $>2$ genomic 
loci, and, 3) a high quantity/quality of DNA content. In this model, the cysts were categorized as mucinous if CEA was $>192 \mathrm{ng} / \mathrm{mL}$ and/or at least one of the three was positive (i.e. abnormal). If none of these four indicators were positive, the cysts were classified as non-mucinous and followed in the model without specific intervention. Of the mucinous cysts, if CEA was $>192 \mathrm{ng} / \mathrm{mL}$ but all three molecular indicators were negative, the cysts were considered to have a predicted benign natural history and followed in the model with imaging surveillance (annually for 3 years, then every 3 years indefinitely). Mucinous cysts with at least one positive molecular indicator were considered borderline/indolent and followed with more frequent imaging surveillance (annually for 5 years and then every 3 years indefinitely); if multiple molecular indicators were positive, then the cyst was considered aggressive and the patient was referred for surgical consultation.

\section{Clinical variables, patient utilities, and costs}

Clinical probabilities including transitional probabilities between different health states and performance characteristics of crosssectional imaging studies, and EUS-FNA with cyst fluid analysis were derived from published information ( Table 1, Table S1). When specific published information was not available, expert opinion was obtained by consensus.

Performance characteristics of PathFinder TG assay were obtained from published data. MEDLINE, EMBASE and Cochrane Databases (January 1977-May 2012) were searched using predefined criteria including the terms "pancreatic cystic neoplasm," "serous," "mucinous," "intraductal papillary mucinous tumor/neoplasm," and "PathFinder TG assay." Abstracts from major gastroenterology meetings from 1997-2012 were also searched for all relevant publications. Manual searches of the bibliography of selected publications were also performed to obtain a baseline and range of sensitivity and specificity estimates of the assay in distinguishing mucinous from non-mucinous cysts and in distinguishing cysts with higher vs. lower malignant potential.

Quality-adjusted life-years (QALY) were estimated by adjusting the life expectancy of each health state by a weight or utility, which reflects patient preferences for that health state [14]. Utility values were derived from published information [15].

\section{Cost estimates}

Costs, not charges, were considered in this analysis ( Table 1, Table S1), and a third-party payer's perspective was taken [1618 ] Only direct costs were considered, and they were adjusted to 2012 US dollars.

\section{Model sensitivity analysis}

As is typical for healthcare economic modeling studies, we ran the model using the baseline estimate for each variable, then tested its robustness (i.e., dependence on assumptions about specific variables) by performing one-way and multi-way sensitivity analyses using a range of performance characteristics obtained from literature ( Table 1, TableS1). Such sensitivity analyses are particularly important when modeling PCNs, because understanding of the natural history of incidental pancreatic cystic lesions, although improved, remains incomplete. The key variables tested were the probabilities of malignancy occurring at initial presentation or during follow-up, cost estimates, and the surgical risk score. A second-order Monte Carlo simulation was also performed for a probabilistic sensitivity analysis in the hypothetical cohort of 1000 patients with incidentally diagnosed solitary pancreatic cystic lesion $[19,20]$

\section{Statistical methods and comparison of outcomes}

To statistically compare the overall cost-effectiveness of the four strategies, we calculated Incremental Cost-Effectiveness Ratio (ICER), and Net Health Benefit (NHB) for each strategy for the same simulated patient cohort $[21,22]$. To analyze the results of the Monte Carlo analysis, relative risk with $95 \%$ confidence intervals and Number Needed to Treat (NNT) were calculated.

\section{Results \\ $\nabla$}

\section{Baseline analysis}

We used the baseline values for all model variables to produce estimates of the average cost per patient and the average survival (QALY) of the simulated patient cohort under each strategy ( Table 2). The baseline analysis showed that Strategy I (Wait \& Watch), was the least expensive at \$19251 per patient but only yielded 10.36 QALY, whereas use of Strategy IV (IMP) was the most effective at balancing cost ( $\$ 19373$ per patient) with increase in QALY (12.33 QALY, a gain of 1.97 QALY over Strategy I). Thus, Strategy IV was considered the most cost-effective among the competing strategies based on the commonly accepted ICER. The current standard of care, Strategy III (EUS + CEA + Cytology), was more expensive than Strategy IV at \$25841 per patient and yielded fewer (11.22) QALY. Strategy II (Resect if operable) yielded the least QALY (9.95) and was also the most expensive ( $\square$ Table 2).

\section{Sensitivity analyses}

One-way sensitivity analysis yielded interesting results: the variables expected to be most important did not significantly affect cost-effectiveness. Such variables included cost of EUS-FNA, cost of PathFinder TG assay, interval of surveillance by imaging, accuracy of cross-sectional imaging in differentiating mucinous from non-mucinous cysts, probability of malignant transformation of benign cysts, and perioperative mortality. When tested across the published range of estimates for percentage of PCNs that are mucinous and percentage of cysts that will progress to malignancy and cost of IMP, Strategy IV continued to be the most cost-effective approach (i.e., highest ICER).

In one-way sensitivity analyses, the surgical risk score did prove to be important in determining the cost and benefit of each management strategy. Between the maximum and minimum risk score, the most cost-effective strategy was Strategy IV (IMP) most often; however, when the risk score was above 8 , the yield in effectiveness in terms of QALY was higher with Strategy I (Wait

\begin{tabular}{|lccc|}
\hline Strategy & Cost (\$) & Effectiveness (QALY) & ICER (\$/QALY) over Strategy I \\
\hline I. Wait \& watch & 19251 & 10.36 & - \\
\hline II. Resect if operable & 32393 & 9.95 & -32054 (Dominated) \\
\hline III. EUS-FNA + Cytology + CEA & 25841 & 11.22 & 6590 (Dominated) \\
\hline IV. Integrated mutational profiling & 19373 & 12.33 & 62 (Preferred) \\
\hline
\end{tabular}

Table 2 Results (using baseline estimates of variables). 
\& Watch). Even using the lowest surgical risk score, Strategy II (Resect if operable) never resulted in the greatest number of QALY gained.

Because the cost of IMP and the performance characteristics of CEA analysis and IMP are likely to be inter-related determinants of the outcomes of the model, we looked at two-way sensitivity analyses by simultaneously varying these probabilities. $\bullet$ Fig. 2 and Fig. 3 show that even when these variables are varied over a broad range of estimates, IMP remains the preferred management method.

\section{Monte Carlo analysis}

A second-order Monte Carlo analysis tests the robustness of the model when all variables randomly assume values across their plausible ranges. We performed this analysis with 1000 distinct hypothetical patients with cystic pancreatic disease using tracking variables to indicate whether surgery was performed or advanced malignancy occurred. Table 3 shows the increase or decrease in per patient cost and QALY for each strategy according to whether the patient went to surgery, or did or did not develop malignant disease. In this Monte Carlo analysis, the number of surgical interventions performed in Strategies I, II, III, and IV were $135,327,247$, and 127 , respectively. Despite the drastic decrease in the number of surgeries from Strategy II to Strategy IV, 23 fewer advanced malignancies occurred in the latter arm while simultaneously reducing the average cost per patient by $\$ 11910$. The current standard of care, Strategy III, allowed advanced malignancy in 19 patients and cost \$5553 more per patient than Strategy IV ( Table 3 ). The number of unresectable malignant cystic tumors diagnosed under each strategy in this cohort was estimated at 18, 32, 19, and 9 for Strategies I, II, III, and IV, respectively.

In the Monte Carlo analysis, relative risk of unresectable pancreatic cyst-adenocarcinoma with Strategy IV (IMP) was 0.18 (95\% $\mathrm{CI}, 0.06-0.53$ ) compared to the nearest competing approach, Strategy III. With this Monte Carlo simulation we arrived at a NNT of 56 (95\%CI 34-120) for Strategy IV. Finally, over a range of commonly used societal Willingness to Pay (WTP) thresholds, Strategy IV yields the highest NHB and Strategy II yields the lowest ( $\bullet$ Fig. 4).

The scatter plots of distribution of ICER of Strategy IV against Strategy III for the simulation trial in the hypothetical cohort show that in nearly $62 \%$ of the simulation trials Strategy IV is dominant; however, in $9.4 \%$ of simulations Strategy IV was inferior being more expensive and yielding a lower ICER. (Fig. 4S).

\section{Discussion}

\section{$\nabla$}

Management of PCNs continues to pose a challenge for clinicians because the malignant potential for any given cyst is difficult to determine. Clinicians can choose surveillance, in which case progression to cancer is a concern, or they can choose surgery which has high associated morbidity. To address this dilemma, several guidelines for management of pancreatic cystic lesions have been published over the last decade $[4,5]$. Unfortunately, given the limitations of current standard diagnostic modalities (e.g., imaging, CEA, cytology), there are many clinical scenarios that cannot be adequately addressed. For instance, guidelines recommend CEA testing to distinguish mucinous from non-mucinous lesions, but CEA has limited utility in assessing malignant potential; recent literature has shown that cysts with CEA lower than
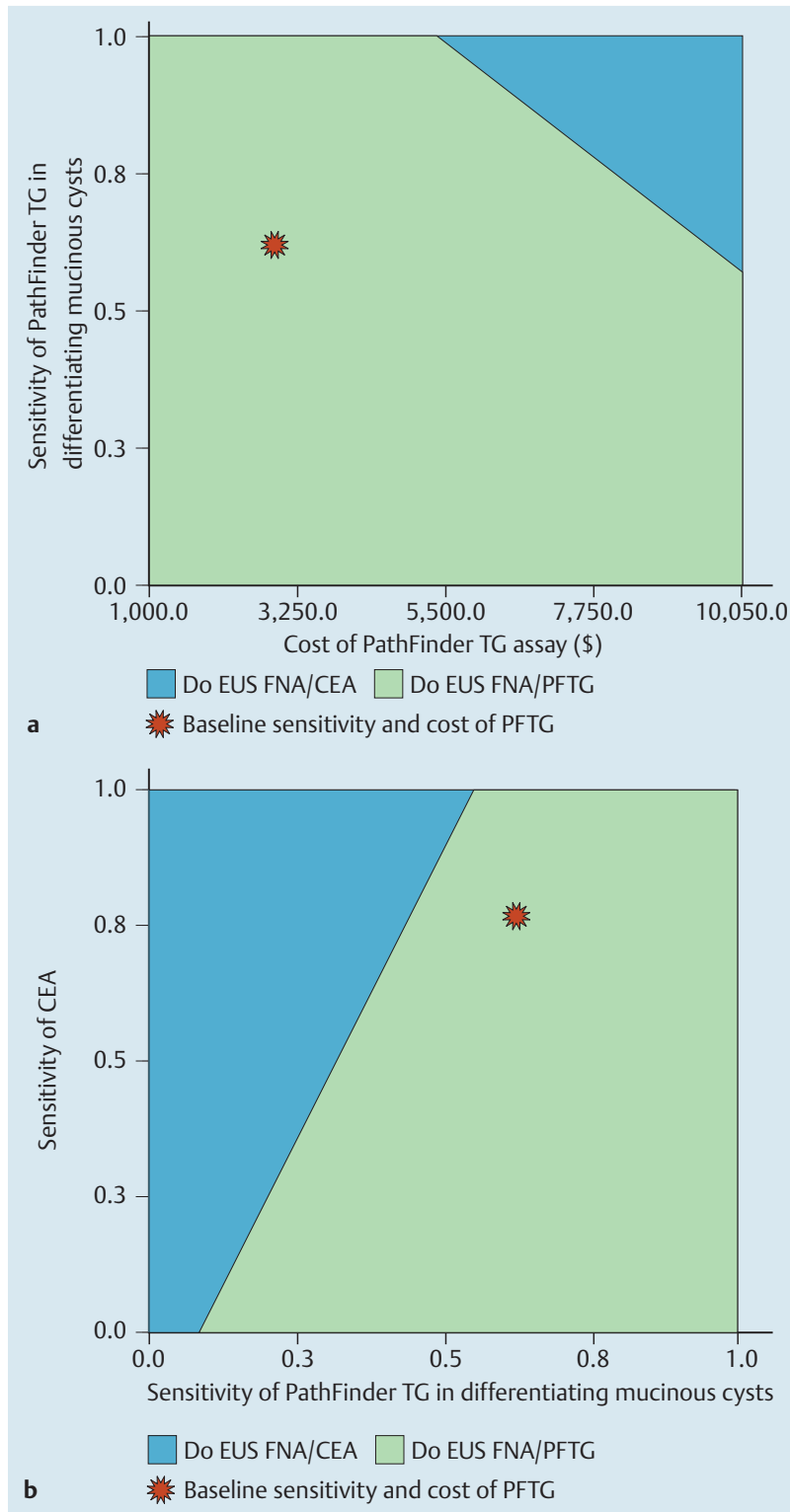

Fig.2 a Results of two-way model sensitivity analysis comparing ranges of cost of integrated mutational profiling (X-axis) vs. diagnostic sensitivity of the PathFinder TG assay in differentiating mucinous from non-mucinous PCNs. For most possible costs and sensitivities, PFTG strategy is preferred (green), except where the cost of PFTG is too high (blue). b Results of twoway sensitivity analysis comparing ranges of sensitivity of PathFinder TG vs. sensitivity of CEA in differentiating mucinous from non-mucinous cysts. PFTG is preferred except in areas marked by blue (low sensitivity of PFTC and relatively high CEA sensitivity). For reference, a 'star' designates the baseline estimates in each nomogram.

the threshold of $192 \mathrm{ng} / \mathrm{mL}$ may be malignant [23,24]. Furthermore, the Sendai guidelines call for resection of mucinous cysts $3 \mathrm{~cm}$ or larger that have concurrent "worrisome features." [4]. However, numerous reports document that cysts smaller than 3 $\mathrm{cm}$ (or even $1 \mathrm{~cm}$ ) may harbor malignancy [23-27]. To address imaging's limitations, the 2012 Sendai guidelines suggest surveillance intervals ranging from every 2 years for cysts $<1 \mathrm{~cm}$ to every 3-6 months for cysts $2 \mathrm{~cm}$ or larger. Similarly, the most recent ACG guidelines (2007) recommend surveillance for cysts strongly suspected to be benign and surgical resection for those strongly suspected to be malignant, but they do not provide specific parameters [5]. In the context of these limitations there 


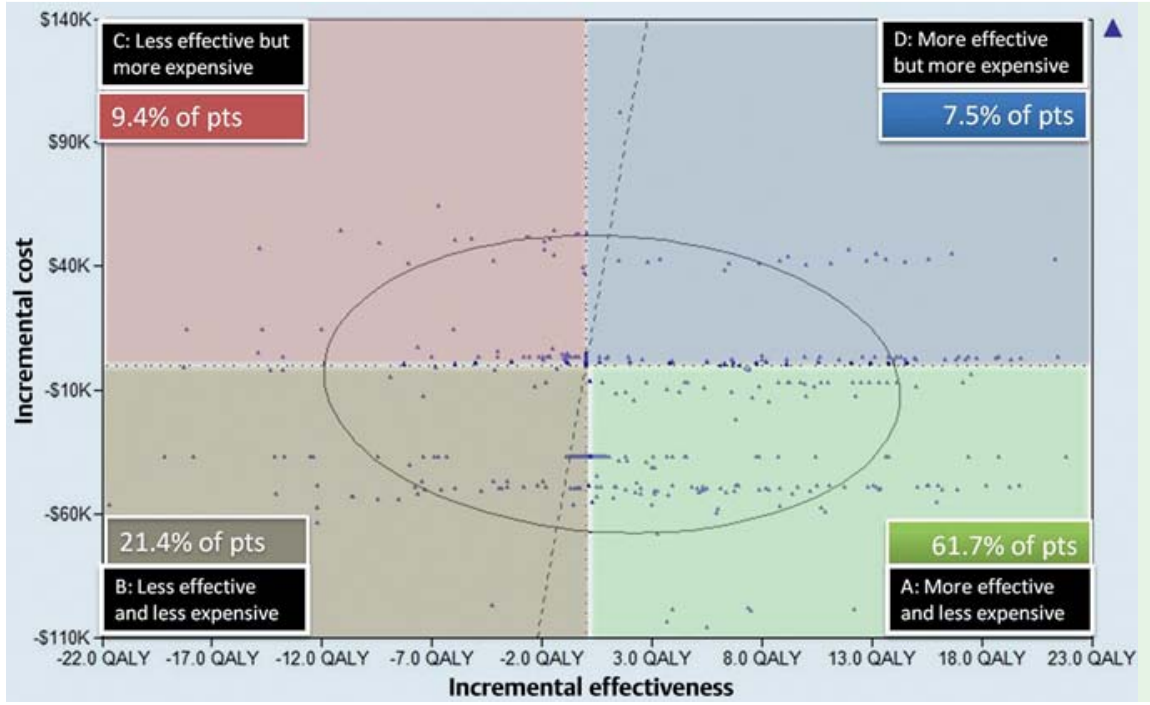

Fig. 3 Results of a Monte Carlo simulation of 1000 patients. Each point represents the increase/ decrease in cost (y-axis) and QALY (x-axis) for a particular patient when choosing Strategy IV over Strategy III.

Table 3 Monte Carlo simulation of frequency of surgery and advanced malignancy with each strategy.

\begin{tabular}{|c|c|c|c|c|c|c|c|c|c|c|c|c|c|}
\hline \multirow[t]{3}{*}{ Surgery } & \multirow{3}{*}{$\begin{array}{l}\text { Advanced } \\
\text { malig- } \\
\text { nancy }\end{array}$} & \multicolumn{12}{|l|}{ Strategy } \\
\hline & & \multicolumn{3}{|c|}{ I Wait \& watch } & \multicolumn{3}{|c|}{ II Resect if operable } & \multicolumn{3}{|c|}{ III EUS + CEA + cytology } & \multicolumn{3}{|c|}{$\begin{array}{l}\text { IV Integrated molecular } \\
\text { pathology }\end{array}$} \\
\hline & & $\begin{array}{l}\text { Patients } \\
\text { (\#) }\end{array}$ & Cost (\$) & QALY & $\begin{array}{l}\text { Patients } \\
\text { (\#) }\end{array}$ & Cost (\$) & QALY & $\begin{array}{l}\text { Patients } \\
\text { (\#) }\end{array}$ & Cost (\$) & QALY & $\begin{array}{l}\text { Patients } \\
\text { (\#) }\end{array}$ & Cost (\$) & QALY \\
\hline Yes & $\mathrm{No}^{1}$ & 135 & 42830 & 14.53 & 327 & 40000 & 12.69 & 247 & 41574 & 13.21 & 127 & 45537 & 13.6 \\
\hline \multirow[t]{2}{*}{ No } & No & 847 & 13150 & 9.77 & 641 & 22596 & 7.92 & 734 & 16518 & 10.64 & 864 & 14118 & 12.22 \\
\hline & Yes & 18 & 109339 & 5.41 & 32 & 106130 & 6.43 & 19 & 111232 & 8.24 & 9 & 113652 & 4.77 \\
\hline Overall & & 1000 & $\$ 18766$ & 10.36 & 1000 & 30876 & 9.95 & 1000 & 24519 & 11.22 & 1000 & 18966 & 12.3 \\
\hline
\end{tabular}

1 The model assumes that surgery prevents progression to advanced malignancy.

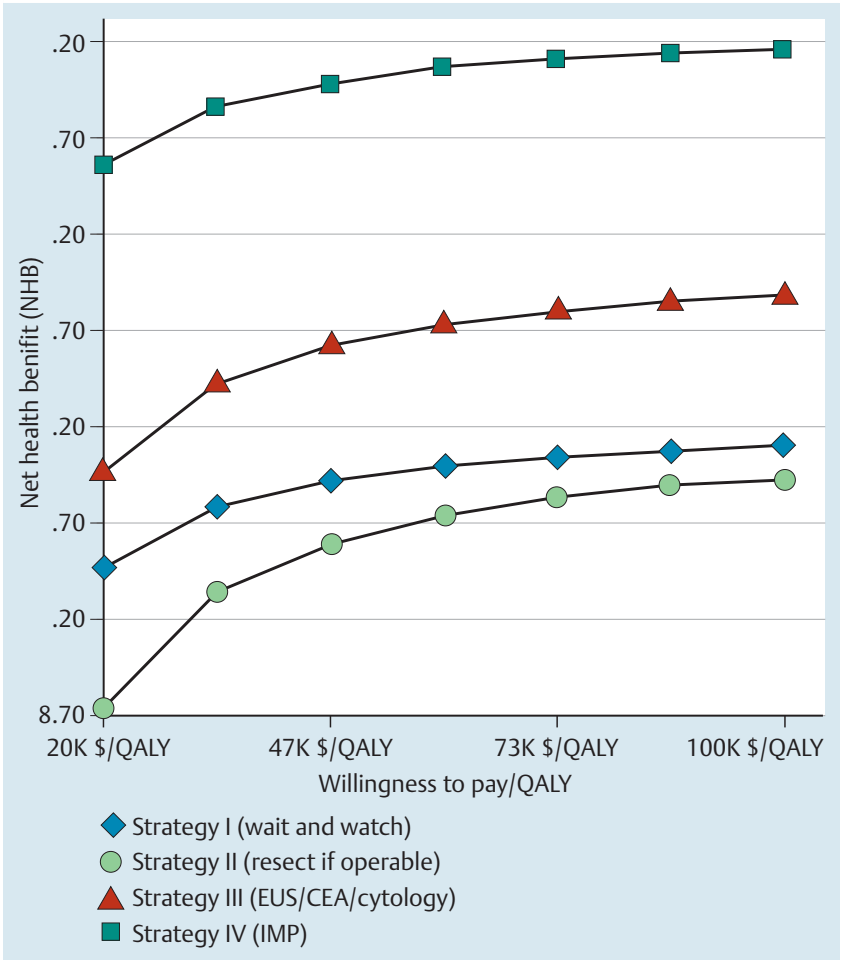

Fig. 4 Average net health benefits (Y-axis) yielded under each strategy against the WTP (X-axis). Strategy IV yields the highest NHB and Strategy II the lowest over a range of Willingness to Pay. is inherent uncertainty in choosing a particular strategy, and scant information exists regarding the cost-effectiveness of different management strategies in this clinical scenario. Given that a controlled, randomized study examining different strategies of managing PCNs with long-term follow-up is unlikely to be available for the future, a practical way to develop management recommendations is to conduct healthcare economic modeling based on available clinical data.

In a previous analysis, we showed that EUS-FNA with cyst fluid analysis for CEA level was the most cost-effective strategy for managing incidental PCNs. This study extended that previous work by adding a management strategy, Strategy IV, which incorporated IMP to determine the malignant potential of cysts; only those patients with "Aggressive" molecular features were referred for surgery, while those with "Benign" or "Statistically Indolent" results were followed with surveillance at progressively longer intervals. Our primary finding was that Strategy IV was very cost-effective compared to the other strategies and provided the greatest increase in QALY. The increase of 2.38 QALY between Strategy II and Strategy IV compares favorably to gains observed in other clinical scenarios, such as use of ablation to eradicate high grade dysplasia in Barrett's esophagus (3.24 QALY increase) [28].

In addition to these findings, our simulation also provided the NNT, a key parameter in assessing cost-effectiveness of management strategies. In this model, PathFinder TG had an NNT of 56, representing the number of patients that needed IMP to prevent advanced malignancy in one patient. As a comparison, the current standard of care for managing PCNs, Strategy III, has an 
NNT of 83, and ablation of non-dysplastic Barrett's esophagus, an emerging practice, has an NNT ranging from $23-250[6,29]$. These data further reinforce the cost-effectiveness of IMP.

Our analyses indicate that the IMP strategy achieves its cost-effectiveness by limiting unnecessary surgery while maintaining the lowest rate of advanced malignancy, thus showing how accurate prediction of malignant potential resolves the dilemma between preventing cancer and performing unnecessary surgery. The high expense of surgery explains why the model was not sensitive to seemingly important variables such as cost of crosssectional imaging or the performance characteristics of IMP; the cost of PathFinder TG testing is greatly exceeded by the expense of even a few unnecessary surgeries.

We acknowledge several limitations of this study, many of which are inherent to any healthcare economic model. Literature on the performance characteristics of current standard diagnostic modalities is abundant; however, as with any newer diagnostic test, available evidence regarding the performance characteristics of IMP is limited. Some of the published studies are limited by sample size and lack of long-term follow-up data. To account for some of the shortcomings of these data and to intentionally bias the model against IMP-based prediction of malignant potential, we used the low end of published estimates of diagnostic accuracy for PathFinder TG for all analyses. Data from the National Pancreatic Cyst Registry, which were not published prior to our search cutoff of May 2012, provide evidence that the estimates used in our model were indeed conservative. IMP sensitivity was over $83 \%$, and specificity was over $90 \%$. The accuracy of IMP was $90 \%$ [12].

Because natural history of PCN are not fully known, the model needed to make assumptions about some variables in which scientifically sound data are lacking; thus, in some cases such assumptions were based on expert opinion [6]. To compensate for this, we used sophisticated techniques of uncertainty analysis, such as second-order Monte Carlo analysis and simulation trials over a wide but biologically plausible range of estimates of important variables to confirm validity of the conclusions. We also assumed low surgical cost and complication rates further biasing the model against IMP. One important limitation is that the surgical risk score developed for this analysis has not undergone formal clinical validation. Another limitation of this study is that it did account for all PCNs. In particular, main duct IPMNs including mixed type (both main and branch duct involvement) were not included because their management would likely involve a different diagnostic algorithm based on endoscopic retrograde cholangiopancreatography, the gold standard for diagnosis of main duct IPMN [30]. Also, we did not consider postoperative morbidity and complications related to EUS-FNA procedures. Finally, only direct costs were taken into account.

While current first-line diagnostic tests have lower cost and wider availability, they cannot provide consistent, meaningful prediction of malignant potential $[23,24]$. Nevertheless, standard clinical management of patients with PCN relies on these firstline tests, the results of which are reflected in Strategy III. Even with the model being heavily biased against IMP, we found that using IMP to predict malignant potential is superior to Strategy III and represents the most cost-effective strategy for managing PCN. These results demonstrate that a reasonably accurate riskstratification tool (e.g., IMP) provides a significant benefit in reducing cost and improving QALY for pancreatic cyst patients.
Competing interests: Ananya Das, M.D.: Research support from Redpath Integrated Pathology for pancreatic cyst healthcare economics prolect and Barrett's esophagus healthcare economics project; Consultant, Boston scientific, Inc., Natick, Massachusetts, USA; Consultant Endostim, Inc., St Louis, MI. William Brugge, M. D.: Research grants from RedPath Integrated Pathology and Asuragen, Girish Mishra, M.D.: Consultant for Novartis Pharmaceuticals, Cook Endoscopy, Pentax Medical. Dennis Smith: Employee, board member, investor, and shareholder at RedPath, Mankanwal Sachdev, M.D.: No conflicts to report. Eric Ellsworth: Employee and shareholder of RedPath.

\section{Acknowledgments}

Rebecca J. Palmer, PhD, funded by RedPath Integrated Pathology (Pittsburgh, Pennsylvania, United States) assisted in the preparation of this manuscript. RedPath provided personnel support to assist with data analysis and interpretation and manuscript preparation.

\section{References}

1 Brugge WR, Lauwers GY, Sahani D et al. Cystic neoplasms of the pancreas. N Engl J Med 2004; 351: 1218-1226

2 Goh BK, Tan YM, Chung YF et al. Pancreatic cysts: a proposed management algorithm based on current evidence. Am J Surg 2007; 193: $749-755$

3 Brugge WR, Lewandrowski K, Lee-Lewandrowski $E$ et al. Diagnosis of pancreatic cystic neoplasms: a report of the cooperative pancreatic cyst study. Gastroenterology 2004; 126: 1330 -1336

4 Tanaka M, Fernandez-del CastilloC, Adsay V et al. International consensus guidelines 2012 for the management of IPMN and MCN of the pancreas. Pancreatology 2012; 12: 183-197

5 Khalid A, Brugge W. ACG practice guidelines for the diagnosis and management of neoplastic pancreatic cysts. Am J Gastroenterol 2007; 102: 2339-2349

6 Das A, Ngamruengphong S, Nagendra $S$ et al. Asymptomatic pancreatic cystic neoplasm: a cost-effectiveness analysis of different strategies of management. Gastrointest Endosc 2009; 70: 690-699

7 Gaujoux S, Brennan MF, Gonen $M$ et al. Cystic lesions of the pancreas: changes in the presentation and management of 1,424 patients at a single institution over a 15-year time period. J Am Coll Surg 2011; 212: $590-600$

8 Fasanella KE, Krasinskas A, Schoedel KE et al. DNA mutational differences in cytological specimens from pancreatic cancer and cholangiocarcinoma. Pancreatology 2010; 10: 429-433

9 Khalid A, Zahid M, Finkelstein SD et al. Pancreatic cyst fluid DNA analysis in evaluating pancreatic cysts: a report of the PANDA study. Gastrointest Endosc 2009; 69: 1095-1102

10 Sawhney MS, Devarajan S, O'Farrel P et al. Comparison of carcinoembryonic antigen and molecular analysis in pancreatic cyst fluid. Gastrointest Endosc 2009; 69: 1106-1110

11 Shen J, Brugge WR, Dimaio CJ et al. Molecular analysis of pancreatic cyst fluid: a comparative analysis with current practice of diagnosis. Cancer 2009; 117: 217-227

12 Al-Haddad MA, Kowalski T, Siddiqui A et al. Integrated molecular pathology accurately determines the malignant potential of pancreatic cysts. Endoscopy 2015; 47: 136-146

13 American Society of Anesthesiologists. 2012 Relative Value Guide Book: a guide for anesthesia values. ASA Publications; 2012

14 Sonnenberg FA, Beck JR. Markov models in medical decision making: a practical guide. Med Decis Making 1993; 13: 322 - 338

15 Klose T. A utility-theoretic model for QALYs and willingness to pay. Health Econ 2003; 12: 17-31

16 Porter GA, Pisters PW, Mansyur C et al. Cost and utilization impact of a clinical pathway for patients undergoing pancreaticoduodenectomy. Ann of Surg Oncol 2000; 7: 484-489

17 Rubenstein JH, Scheiman JM, Anderson MA. A clinical and economic evaluation of endoscopic ultrasound for patients at risk for familial pancreatic adenocarcinoma. Pancreatology 2007; 7: 514-525 
18 Weinstein MC, Siegel JE, Gold MR et al. Recommendations of the Panel on Cost-effectiveness in Health and Medicine. JAMA 1996; 276: $1253-1258$

19 Doubilet $P$, Begg CB, Weinstein MC et al. Probabilistic sensitivity analysis using Monte Carlo simulation. A practical approach. . Med Decis Making 1985; 5: $157-177$

20 Gold M, Siegel J, Russell L, Weinstein MC (eds.). Cost-Effectiveness in Health and Medicine. New York, NY: Oxford University Press; 1996

21 Heitjan DF. Fieller's method and net health benefits. Health Econ 2000; 9: $327-335$

22 Stinnett AA, Mullahy J. Net health benefits: a new framework for the analysis of uncertainty in cost-effectiveness analysis. Med Decis Making 1998; $18: 68-80$

23 Nagula S, Kennedy T, Schattner MA et al. Evaluation of cyst fluid CEA analysis in the diagnosis of mucinous cysts of the pancreas. J Gastrointest Surg 2010; 14: 1997-2003

24 Kucera S, Centeno BA, Springett $G$ et al. Cyst fluid carcinoembryonic antigen level is not predictive of invasive cancer in patients with intra- ductal papillary mucinous neoplasm of the pancreas. JOP 2012; 13 : 409-413

25 Fritz S, Klauss M, Bergmann Fet al. Small (Sendai negative) branch-duct IPMNs: not harmless. Ann Surg 2012; 256: 313-320

26 Lee CJ, Scheiman J, Anderson MA et al. Risk of malignancy in resected cystic tumors of the pancreas $\leq 3 \mathrm{~cm}$ in size: is it safe to observe asymptomatic patients? A multi-institutional report. J Gastrointest Surg 2008; 12 : 234-242

27 Sahani DV, Saokar A, Hahn PF et al. Pancreatic cysts $3 \mathrm{~cm}$ or smaller: how aggressive should treatment be? Radiology 2006; 238: 912 - 919

28 Inadomi JM, Somsouk M, Madanick RD et al. A cost-utility analysis of ablative therapy for Barrett's esophagus. Gastroenterology 2009; 136 : $2101-2114(\mathrm{e} 1-6)$

29 Ganz RA. Barrett's esophagus. N Engl J Med 2010; 362: 1448 - 1449 author reply 1449

30 Gourgiotis S, Germanos S, Ridolfini MP. Presentation and management of pancreatic cystic neoplasms. J Clin Gastroenterol 2007; 41: 599608

\section{Supplemental Figures and Tables:}

Fig. S1, S2, S3 S4, Table S1, S2, S3

supplementary content viewable at:

http://dx.doi.org/10.1055/s-0034-1392016 